\title{
An instrumented saxophone mouthpiece and its use to understand how an experienced musician play.
}

\author{
Ph. Guillemain, Ch. Vergez, D. Ferrand ${ }^{\text {a) }}$ and A. Farcy \\ Laboratoire de Mécanique et d'Acoustique, CNRS UPR 7051 \\ 31 Chemin Joseph Aiguier, 13402 Marseille Cedex 20, France
}

(Dated: 8 janvier 2010)

\begin{abstract}
An instrumented saxophone mouthpiece has been developed to measure, during the player's performance, the evolution of important variables : the mouth pressure, the mouthpiece pressure and the force applied on the reed by the lower lip. Moreover, according to the pressure signals in the mouth and in the mouthpiece, the instantaneous ratio of the vocal tract input impedance and of the saxophone input impedance is estimated at frequencies multiple of the playing frequency (using the concept of Gabor mask). On the selected sound examples, analyses reveal many aspects of the strategies of the player. First of all, the role of the vocal tract in the characteristics of the sound production is sometimes prominent. Secondly, the sound production on the desired note (and register) as well as pitch correction seem to be the result of complementary adjustments of the mouth pressure and of the lip pressure on the reed. This is not in agreement with musicians feeling, since they often claim to let their force on the reed unchanged during the note and from note to note.
\end{abstract}

PACS numbers: 43.75.Ef, 43.75.Pq, 43.75.Yy

\section{INTRODUCTION}

Among self sustained musical instruments, one can find those for which the instrument maker leaves the highest number of degrees of freedom to the player. Among this family, woodwind instruments have a specificity : The player, thanks to his vocal tract and his lip control on the reed, is part of the whole resonator involved in the functioning of the instrument. Therefore, it is particularly interesting to be able to measure how the player controls his instrument and what are the consequences of its gestures on the regimes and the sound produced. This general subject has been tackled by many authors aiming at understanding the role of the musician ${ }^{3,5,7,11}$, with studies devoted to the role of the vocal tract in the sound production ${ }^{1,2,4,10,18,19}$, or to the lip control to produce vibratos ${ }^{12}$.

In this paper, an instrumented saxophone mouthpiece and signal processing tools, developed to gather information about the playing, are first presented. They are used to inspect the strategies of an experienced player concerning the use of the vocal tract and the lip pressure. The methodology used here involves the estimation of the ratio between the input impedance of the vocal tract and that of the saxophone.

The paper starts with the presentation of the experimental device. It aims at measuring simultaneously the mouth pressure, the mouthpiece pressure and the lip

\footnotetext{
a) Electronic address: guillemain, vergez, ferrand@lma.
} cnrs-mrs.fr pressure on the reed. The device is made of two probes and pressure sensors in order to collect the mouth and mouthpiece pressures and an FSR sensor to measure the lip pressure on the reed. Then, the classical physical hypotheses allowing to measure the impedances ratio between the vocal tract and the instrument at harmonic frequencies of the note played, are briefly recalled. The description of the experimental device is followed by the presentation of the signal processing tools, based on the concept of Gabor masks. After recalling some definitions, it is shown how it can be used to obtain and represent a time-varying transfer function between two non-stationary multi-components signals sharing the same frequency content. It is a key-point in order to estimate the time-varying impedances ratio.

Section III presents the various experiments that were conducted with an experienced jazz saxophone player. The first examples investigate how he uses his vocal tract to produce effects such as pitch bend and glissando. Results are justified thanks to the use of a simple input impedance model of an alto saxophone. The last examples illustrate how both the vocal tract and the lip pressure are used on chromatic scales in order to facilitate the emission of the right note with the right pitch.

Last section is devoted to conclusions and perspectives of this work. 

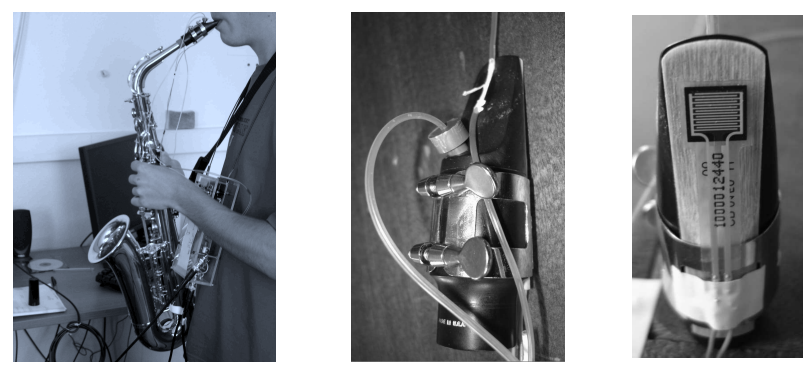

FIG. 1. Left : saxophone used for the experiments, with flexible probes to measure mouth and mouthpiece pressure, as well as the electronic board hanging from the neck of the player ; center : close view with one probe inserted in the mouthpiece and one probe to fit behind the upper teeth; right : close view on the reed showing the FSR sensor.

\section{EXPERIMENT SETUP AND POST-PROCESSING}

\section{A. Experimental setup}

\section{Description of the experimental setup}

The experimental set-up is visible on figure 1 : it consists mainly of a saxophone mouthpiece equipped with two types of sensors :

- a FSR (Force Sensitive Resistance IEE FSR150) sensor to monitor the pressure applied on the reed by the player's lower lip.

- two air pressure piezo-resistive sensors to measure both mouth pressure and moutpiece pressure.

- an additional microphone (B\&K 4133) is placed at $50 \mathrm{~cm}$ of the bell, in order to record the external pressure.

For these experiments, the saxophone used is an alto Yamaha YAS-275, and the instrumented mouthpiece is a Meyer 7 medium chamber with a medium Fibracell reed. Pressure sensors were deported at the end of cylindrical flexible probes. This choice was motivated by two reasons : firstly because the mouth is a humid area, and secondly in order to avoid possible mechanical coupling between the sensor inserted in the mouthpiece rigid body and bore vibrations (due to the coupling with the acoustic field or to reed hits on the lay). These probes have been chosen and calibrated carefully (see section II A 3).

The electronics for the conditioning of all three sensors (two pressure sensors and the FSR) as well as the alimentation are packed on a small card worn by the saxophonist, as shown on figure 1 (left picture). To avoid additional cables, a $9 \mathrm{~V}$ battery has been mounted on the board. The whole system is then connected by BNC cables to a real time acquisition system (dSpace/Matlab) : 16 bits ADC (Analog to Digital Converters), $40 \mu s$ conversion time (hence corresponding to a sampling frequency $\left.f_{s}=25 k H z\right)$.

\section{Details concerning the sensors}

Precise details are given in this section concerning the electronic implementation in order to make it easy to reproduce the same experiment.

a. FSR sensor: The FSR150 sensor (Manufacturer I.E.E.) is a force sensor whose data-sheet specifies a rising time $t_{r}<2 m s$ and a repeatability error $\epsilon_{r}<2 \%$. When applying a pressure on the force sensor, the internal resistance $R_{\mathrm{FSR}}$ decreases due to the piezo-resistive effect in the semiconductor embedded in the sensor. The output voltage signal is obtained by a simple resistance (cf. figure 2).

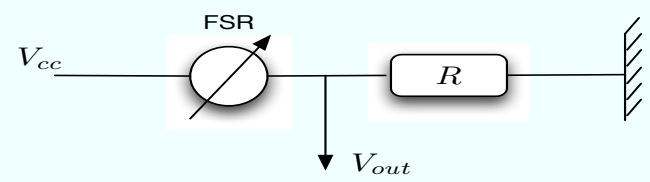

FIG. 2. Electrical conditioning for the FSR : the voltage $V_{\text {out }}$ is provided through a series resistance $R=39 k \Omega$.

According to this simple interface, the voltage provided by the electronics $\left(V_{\text {out }}\right)$ is :

$$
V_{\text {out }}=\frac{R}{R+R_{\mathrm{FSR}}} V_{c c}
$$

where $V_{c c}$ is the $5 V$ voltage supply provided by a battery and a $5 V$ regulator, $R$ is set up at $39 \mathrm{k} \Omega$ and $R_{f}$ is the variable resistance of the FSR. The choice of the resistance $R$ has been done to get an almost complete $5 \mathrm{~V}$ full-scale operation from no weight and maximum weight applied on the reed (around 900g).

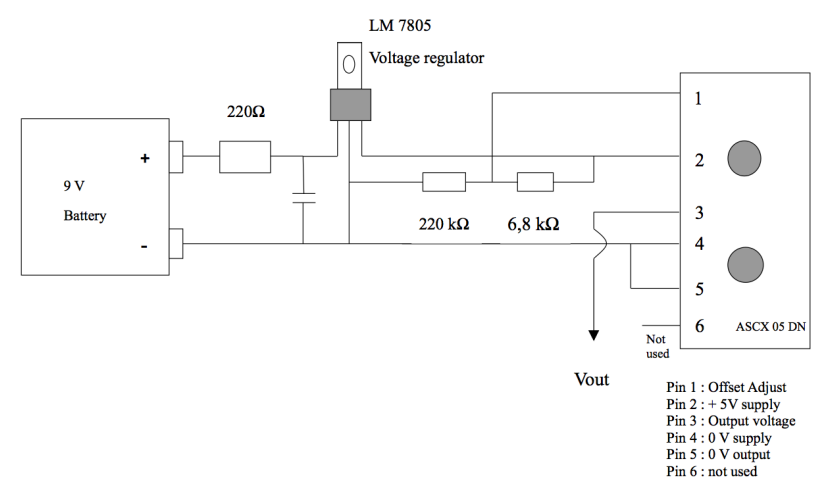

FIG. 3. Electrical mount for the sensor used to measure the mouth pressure. Thanks to an offset on pin 1, the useful range has been maximized. 
b. Pressure sensors : The two pressure sensors used for the experiments are piezo-resistive ASCX05 DN, i.e. with a range of 5 Psi differential pressure $(5 \mathrm{Psi} \simeq 35 \mathrm{kPa})$. These devices are characterized for operation from a single $5 \mathrm{~V}$ dc-supply. For this purpose, a $9 \mathrm{~V}$-battery with a voltage regulator (LM -7805) provide a very stable voltage supply (which is light enough to be mounted on the portable board). The time response specified in datasheet is about $100 \mu \mathrm{s}$. The pressure sensors as well are fixed on the electronics board.

The pressure sensor dedicated to the mouth pressure measurement provides a voltage which is always positive, due to an offset voltage applied on its first pin (see figure 3 ). This has been done in order to have a maximum useful range. For the mouthpiece pressure, since we measure a signal centered around 0 (acoustic pressure), no offset is applied since the pressure sensor yields a voltage of $2.3 \mathrm{~V}$ without differential pressure applied on it in order to operate for differential pressure over the $4.5 \mathrm{~V}$ full scale range.

\section{Calibration}

a. FSR sensor: This sensor has been calibrated statically using small weights (from $10 \mathrm{~g}$ up to a maximum of $900 \mathrm{~g}$ ), exerting a known force perpendicularly on the FSR. Results when increasing or decreasing the force on the FSR reveal moderate hysteresis (see figure 4, left, dotted lines). On the same picture are also plotted (plain lines) the results of other measurements when the force is increased, which show that the repeatability error is of the same order as the hysteresis.

One should notice that the incertitude on the measured force is also yielded by the actual position of the lower lip on the sensor. Therefore, some measurements have also been done few millimeters off-center of the sensitive area $(+/-1 \mathrm{~mm})$. Results are presented in figure 4 (right picture).
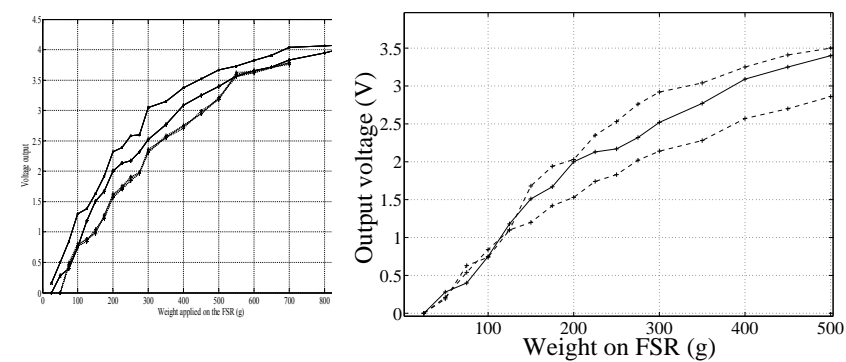

FIG. 4. FSR static calibration curve to estimate both hysteresis and repeatability errors (left) as well as errors due to a misalignment between the lip and the FSR (right) : 3 positions on and off axis on the sensible area $(-1,0,+1) \mathrm{mm}$.

Errors are of the same order of magnitude. Therefore, since the position of the player's lower lip cannot be guaranteed, repeatability or hysteresis errors should not be considered as precluding for the measurement, which in any way will be exploited in the following in a qualitative way.

b. Pressure sensors : ASCX sensors are sold calibrated for span to within maximum $1 \%$ (typically $0.2 \%$ ) of full-scale operation, and temperature compensated over a range of $0^{\circ} \mathrm{C}$ to $70^{\circ} \mathrm{C}$. The sensitivity of each sensor has been checked by comparison with results given by a manometer. They are found to be $9.1 k \mathrm{~Pa} / \mathrm{V}$ (resp. $9.27 \mathrm{kPa} / \mathrm{V}$ ) for the sensor used in the mouthpiece (resp. in the mouthpiece) and both of them display a linear characteristics, as seen in figure 5 .
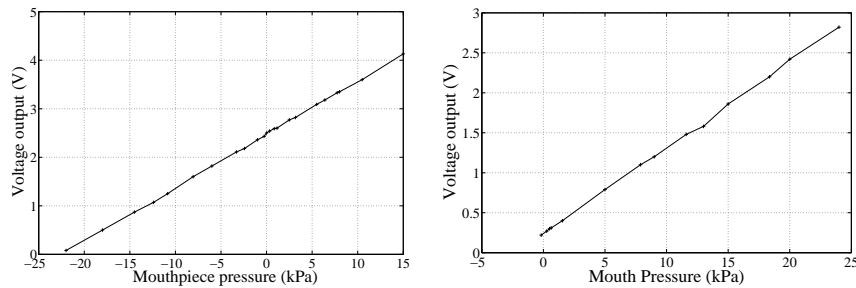

FIG. 5. Estimation of the sensitivity of each pressure sensor : mouthpiece pressure sensor (left), mouth pressure sensor (right).

c. Probes : It is obvious that pressure signals measured at the outlet of the probes are different from pressure signals at their inlet. These differences depend on the probes geometry that has hence been chosen carefully and characterized experimentally. Indeed both diameter and length of the cylindrical probes have been chosen through an iterative process with the aim to :

- have a pressure transfer function between the inlet and the outlet as close as possible for the two probes (lengths of each probe are adjusted to achieve this goal). The motivation will become clear in section II B 1.

- keep a bandwidth sufficiently large for measurements (typically $2 k H z$ ). This supposes a sufficiently large probe diameter.

- minimize the disturbance of the input impedance of the probe inserted in the mouthpiece on the global input impedance. This implies a sufficiently small diameter, since the bore of the saxophone and the probe are mounted in parallel.

The two latter points appear contradictory and in order to find a compromise, simulations have been carried out. A good compromise is found with probes of length $50 \mathrm{~cm}$ and diameter $0.6 \mathrm{~mm}$. In figure 6 we present the input impedance of a saxophone (corresponding to $C \sharp 3$ fingering, the lowest note of the instrument) as well as the one with the same saxophone with that probe (diameter $0.6 \mathrm{~mm}$, length $0.5 \mathrm{~m}$ ) mounted in parallel. Maximum influence is located at the four first impedance maxima with differences of $7 \%, 8 \%, 7 \%$ and $5 \%$ respectively. No frequency 


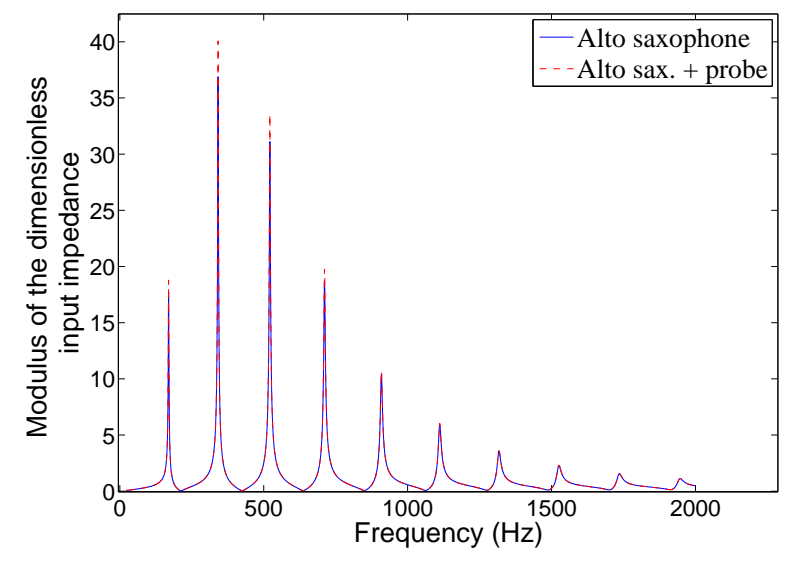

FIG. 6. Simulation of the influence of the probe (diameter $0.6 \mathrm{~mm}$, length $0.5 \mathrm{~m}$ ) on the input impedance of the saxophone.

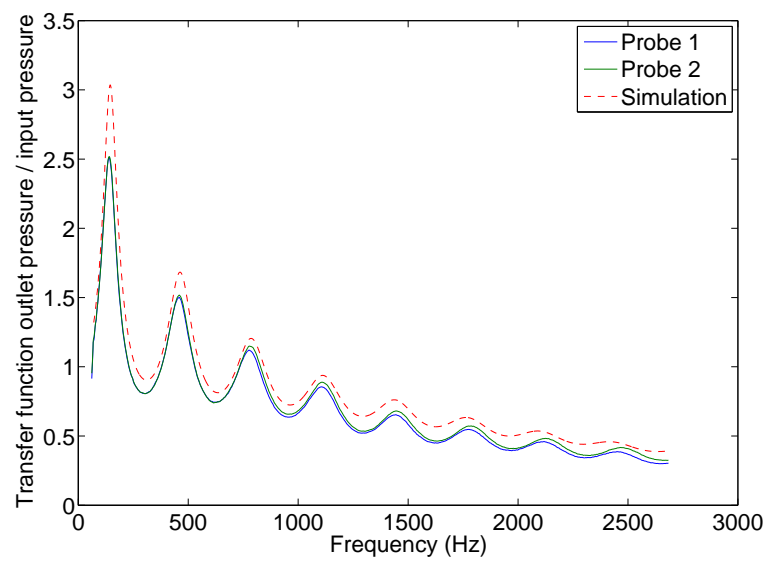

FIG. 7. Simulation of the pressure (inlet/outlet) transfer function for the chosen probes, and their measurement. Probes dimensions : diameter $0.6 \mathrm{~mm}$, length $0.5 \mathrm{~m}$.

deviation is observed. This has been done for all the fingerings of the first register. For those simulations (based on $^{6}$ and $^{16}$ ), the admittance of the sensor at the end of the probe, as well as the potential leaks are ignored and hence the boundary condition is assumed to be perfectly rigid.

Once the probes have been chosen through the simulation process, their pressure transfer functions are measured and compared to the simulations (see figure 7). For the simulations, the transfer function between the inlet and the outlet of the probe is $P_{o} / P_{i}=1 / \cos (k l)$ (where $k$ is the wave number and $l$ is the probe length) the first peak of which being at $170 \mathrm{~Hz}$, as observed on figure 7 .

\section{Position of the pressure sensors}

Through our own investigations, we concluded that :

- Time-frequency plots for sustained notes are not si-

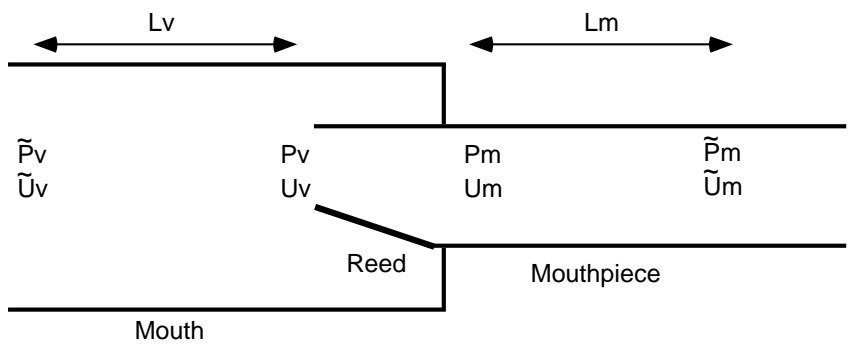

FIG. 8. Sketch of the experiment and physical variables.

gnificantly affected by the position of the probe inside the mouth. It is therefore placed, for ergonomic reasons, behind the upper teeth.

- The position of the probe in the mouthpiece is more a question of compromise : the distance with the other probe should be minimized (see section II B 1) but due to the jet-induced turbulence, it has to be kept downward the mouthpiece entrance. Following ${ }^{20}$, who chose a position at $39 \mathrm{~mm}$ downward the tip of the mouthpiece, and in accordance with our own tests to check the evolution of the signal to noise ratio, the probe is inserted at $40 \mathrm{~mm}$ downward the tip.

\section{B. Estimate of input impedances ratio between the vocal tract and the saxophone}

\section{Principle}

Figure 8 shows a sketch of the experiment and the physical variables. Let $Z_{m}(\omega)$ and $Z_{v}(\omega)$ denote respectively the acoustic impedances of the vocal tract and the instrument, seen from the reed :

$$
\frac{P_{m}(\omega)}{U_{m}(\omega)}=Z_{m}(\omega), \quad \frac{P_{v}(\omega)}{U_{v}(\omega)}=-Z_{v}(\omega)
$$

Assuming acoustic flow conservation ${ }^{18,20}$ between the mouth and the mouthpiece $\left(U_{m}(\omega)=U_{v}(\omega)\right)$, it yields :

$$
\frac{P_{v}(\omega)}{P_{m}(\omega)}=-\frac{Z_{v}(\omega)}{Z_{m}(\omega)}
$$

which means that the ratio of the impedances is the transfer function between the mouth and mouthpiece pressures.

Due to experimental constraints, the actual measurement points of the pressures are located at distances $L_{v}$ and $L_{m}$ of the reed. Using the transmission line formalism, the pressures $\tilde{P}_{v}(\omega)$ and $\tilde{P}_{m}(\omega)$ at the measurement points are :

$$
\begin{aligned}
\tilde{P}_{v}(\omega) & =\cos \left(k_{v} L_{v}\right) P_{v}(\omega)+j Z_{v} \sin \left(k_{v} L_{v}\right) U_{v}(\omega) \\
\tilde{P}_{m}(\omega) & =\cos \left(k_{m} L_{m}\right) P_{m}(\omega)-j Z_{m} \sin \left(k_{m} L_{m}\right) U_{m}(\omega)
\end{aligned}
$$

where $Z_{v}$ and $Z_{m}$ are the characteristic impedances, depending on the cross-sections of the mouth and the mouthpiece and $k_{v}, k_{m}$ are the wave-numbers. 
From the system of equations (4), it yields :

$$
\frac{\tilde{P}_{v}(\omega)}{\tilde{P}_{m}(\omega)}=-\frac{\cos \left(k_{v} L_{v}\right) Z_{v}(\omega)-j Z_{v} \sin \left(k_{v} L_{v}\right)}{\cos \left(k_{m} L_{m}\right) Z_{m}(\omega)-j Z_{m} \sin \left(k_{m} L_{m}\right)}
$$

Equation (5) shows that equation (3) is a low frequency approximation, assuming that $L_{v}$ and $L_{m}$ are small.

\section{Gabor transforms and mask}

Here, the signals are processed using time-frequency methods, that will allow to analyze both signals and estimate and calculate the time varying transfer function of Eq. (??). The concept of Gabor mask ${ }^{9}$ is used and is now briefly recalled and illustrated on a simulated example.

Let us denote respectively by $s_{v}(t), s_{m}(t)$ the signals measured in the mouth and in the mouthpiece and $L_{v}(\tau, \alpha), L_{m}(\tau, \alpha)$ their Gabor transforms defined by :

$$
L_{v, m}(\tau, \alpha)=\int s_{v, m}(t) W(t-\tau) \exp (-j \alpha(t-\tau)) d t
$$

where $W$ is a (real, symmetric) gaussian localization window and $\tau, \alpha$ respectively represent sampled subsets of the continuous time and frequency axes of the representation, chosen to insure that the window $W$ generates a tight Gabor frame ${ }^{9}$. The time-frequency transfer function, called Gabor mask $M(\tau, \alpha)$ is defined as the time frequency function allowing to build $L_{v}(\tau, \alpha)$, assuming that $L_{m}(\tau, \alpha)$ is known :

$$
L_{v}(\tau, \alpha)=M(\tau, \alpha) L_{m}(\tau, \alpha)
$$

It can be shown? that a stable estimator of $M(\tau, \alpha)$ is :

$$
M(\tau, \alpha)=\frac{L_{v}(\tau, \alpha) \bar{L}_{m}(\tau, \alpha)}{C+\left|L_{m}(\tau, \alpha)\right|^{2}}
$$

where the bar denotes the complex conjugate and $C$ is a small adjustable regularization constant that insures the stability of the inversion when $\left|L_{m}(\tau, \alpha)\right|$ is close to zero.

In order to demonstrate the relevancy of the use of the Gabor mask to estimate time-varying transfer functions on time varying signals, let us consider two signals of the form :

$$
s_{v, m}(t)=A_{v, m}(t) \exp \left(j\left(\phi(t)+\phi_{v, m}\right)\right)
$$

where $A_{v, m}(t)>0$. Here, it is assumed that the two signals share the same time-varying partials up to constant phases $\phi_{v, m}$. Under asymptotic hypotheses $\left(A_{v, m}(t)\right.$ and $d \phi(t) / d o t$ are slowly varying on the time support of the window $W(t)$ ), the Gabor transforms of the signals can be approached by ${ }^{15}$ :

$$
L_{v, m}(\tau, \alpha) \simeq A_{v, m}(\tau) \hat{W}\left(\phi^{\prime}(\tau)-\alpha\right) \exp \left(j\left(\phi(\tau)+\phi_{v, m}\right)\right)
$$

where $\hat{W}$ denotes the (real) Fourier transform of $W$ and $\phi^{\prime}$ denotes the time derivative of $\phi$. According to equation
(10), the Gabor transforms show a frequency localization (along the $\alpha$ axis) given by the Fourier transform of the window centered along the curve that describes the frequency modulation law of the signal (corresponding to : $\phi^{\prime}(\tau)=\alpha$ ) and are modulated in amplitude by $A_{v, m}(\tau)$.

From equations (10) and (8), the Gabor mask is given by :

$$
M(\tau, \alpha)=\frac{A_{v}(\tau) A_{m}(\tau) \hat{W}\left(\phi^{\prime}(\tau)-\alpha\right)^{2} \exp \left(j\left(\phi_{v}-\phi_{m}\right)\right)}{C+A_{m}(\tau)^{2} \hat{W}\left(\phi^{\prime}(\tau)-\alpha\right)^{2}}
$$

For non zero values of $\hat{W}\left(\phi^{\prime}(\tau)-\alpha\right)$ (i.e for values of $\alpha$ in the vicinity of $\left.\phi^{\prime}(\tau)\right)$, up to the regularization constant $C$, the modulus of $M(\tau, \alpha)$ shows a constant value along the $\alpha$ axis, modulated by the ratio $A_{v}(\tau) / A_{m}(\tau)$ along the $\tau$ axis.

The signals considered here are supposed to be harmonic : $s_{v, m}(t)=\sum_{k=1}^{N} A_{v, m}^{k}(t) \exp \left(j k \phi(t)+\phi_{v, m}^{k}\right)$. In this case, the bandwidth of $W$ must be adapted so that $\hat{W}\left(k \phi^{\prime}(\tau)-l \phi^{\prime}(\tau)\right)$ can be considered negligible for $k \neq l$. This condition insures the time-frequency independence of the Gabor transforms corresponding to each harmonics and allows to interpret the Gabor mask as a sampled version (along the frequency axis, at the rate of the fundamental frequency) of the time varying transfer function.

The middle panel of figure 9 show the modulus of the Gabor transform of a one second simulated harmonic signal $s_{m}(t)$. The components are frequency modulated with a sinusoidal vibrato. Their amplitudes are decaying exponentially with respect to the time and are modulated with a sinusoidal tremolo. The top panel shows the modulus of the Gabor transform of a time varying filtered version $s_{v}(t)$ of the harmonic signal $s_{m}(t)$. The filter chosen in this example is a fourth order bandpass, whose resonance frequency, bandwidth and gain are increasing with respect to the time. The bottom panel shows the modulus of the Gabor mask, computed according to the equation (8). The three features of the filter mentioned above are clearly visible.

Figure 10 shows superimposed the modulus of the theoretical transfer function of the time varying filter, whose coefficients have been fixed to their values at $t=0.5 \mathrm{~s}$ in solid line, and a vertical slice of the modulus of the Gabor mask at $t=0.5 \mathrm{~s}$ in dotted line. The good agreement between the theoretical filter and the estimated filter shows that the Gabor mask concept seems to be a suitable tool to characterize quantitatively the time varying transfer function between the mouth pressure and the mouthpiece pressure in performance situation.

\section{Discussion : sampled input impedance and possible misinterpretations}

Though equation (3) looks simple, the attention of the reader is drawn on possible misinterpretations of the results. In particular, in permanent regime, the only quantity that remains measurable is the pressures ratio at the harmonics of the playing frequency. Independently of the 


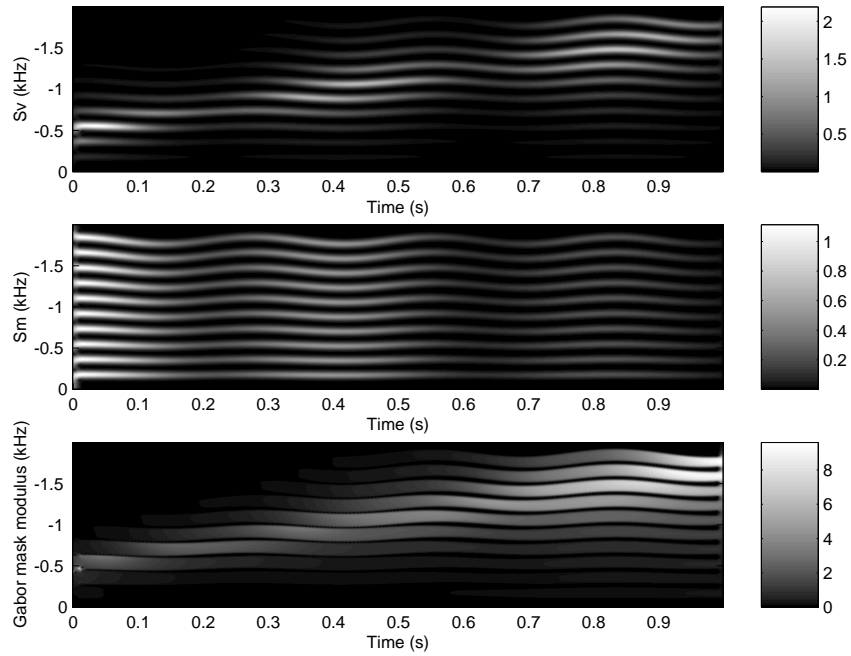

FIG. 9. Top panel : Gabor transform of simulated $s_{v}$; Middle panel : Gabor transform of simulated $s_{m}$; Bottom panel : Gabor mask. Horizontal axes in s, vertical axes in $\mathrm{kHz}$.

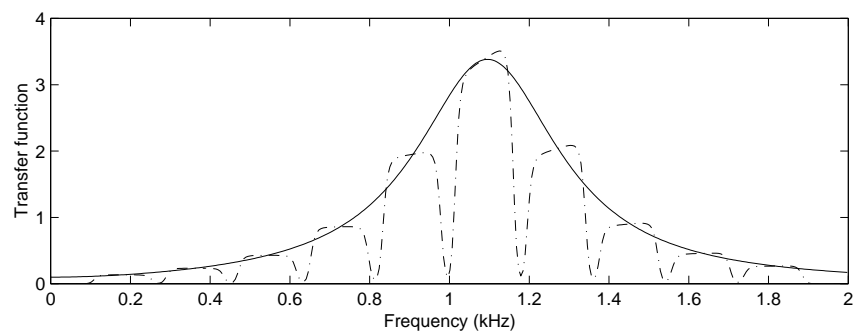

FIG. 10. Solid line : Theoretical transfer function of the filter at $t=0.5 \mathrm{~s}$. Dotted line : Vertical slice of the Gabor mask at $t=0.5 s$. Horizontal axis in $\mathrm{kHz}$.

vocal tract, the level of the harmonics of the mouthpiece pressure is related to a sampled version of the bore impedance at multiples of the fundamental frequency, hence modified by the inharmonicity of the bore impedance peaks (see figure 11). Therefore, even if fingerings are let unchanged, a modification of the impedance ratio should not be systematically interpreted as a modification of the vocal tract acoustic impedance. This is a key point since the sampling of the instrument input impedance is tremendously altered as the playing frequency is changed.

This is illustrated through simulation (see figure 11). The impedance model used here to help the interpretation of the results corresponds to that discussed e.g. by Dalmont ${ }^{6}$ :

$$
Z_{e}(\omega)=\frac{1}{\frac{1}{j \tan (k L)}+\frac{1}{j k x_{e}}+\frac{j k x_{e}}{3}}
$$

In this model, a simple volume is mounted in parallel with a truncated cone. $L$ represents the length of the cone, $x_{e}$ represents the length of the missing part of the cone and $k=k(\omega)$ is the wave-number including viscothermal losses.
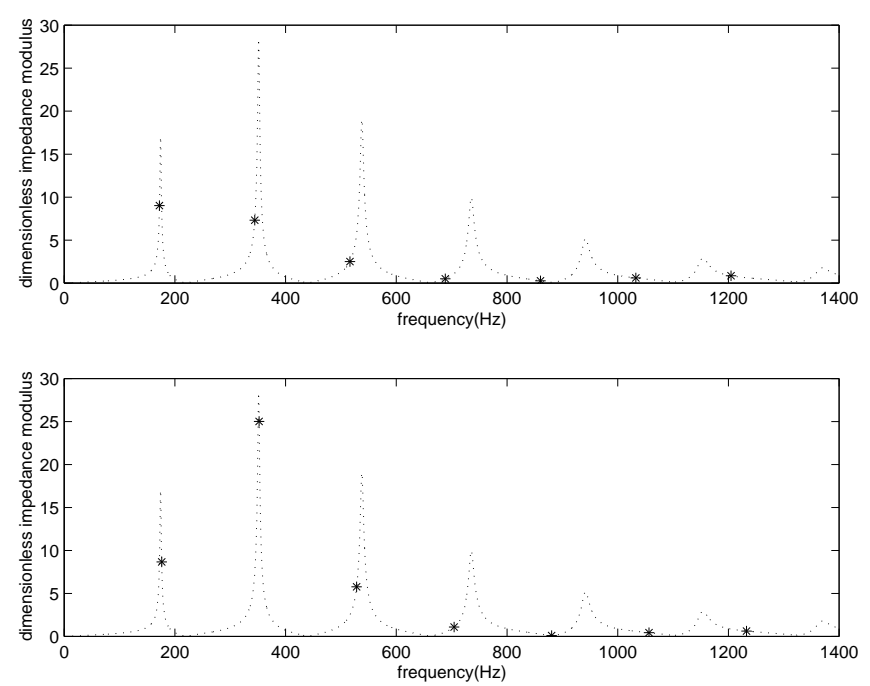

FIG. 11. Typical saxophone input impedance (F3 piano, fundamental frequency $174 \mathrm{~Hz}$ ). The stars correspond to the sampled values of the impedance modulus at frequencies strictly harmonic of $172.1 \mathrm{~Hz}$ (top) and $176.1 \mathrm{~Hz}$ (bottom).

The term $j k x_{e} / 3$ models the mouthpiece whose volume corresponds exactly to that of the missing part of the cone and ideally compensates the inharmonicity of the impedance peaks of the truncated cone at low frequency. This particular mouthpiece volume is derived from the continuous fraction expansion of $1 /\left(j \tan \left(k x_{e}\right)\right) \simeq 1 /\left(j k x_{e}\right)+j k x_{e} / 3$ for small values of $k x_{e}$.

In figure 11, the simulated input impedance is fully defined with the following geometrical parameters : The input radius of the cone is $r=6 \mathrm{~mm}$. The top angle is $\theta=$ $3.36^{\circ}$. The length is $L=0.755 \mathrm{~m}$. These parameters lead to a maximum of the first impedance peak at $174.1 \mathrm{~Hz}$. These parameters correspond to the glissando example depicted in subsection III C.

The stars correspond to the sampled values of the impedance modulus at frequencies strictly harmonic of $172.1 \mathrm{~Hz}$ (top) and $176.1 \mathrm{~Hz}$ (bottom). Since the impedance peaks are not in perfect harmonic relationships, it can be observed on the upper picture that the sampled impedance is smaller for the second peak than for the first one, while on the bottom picture it is the opposite. Moreover, it can be observed on both pictures that after the third peak, the sampled impedances are very small since the stars fall in impedance minima.

\section{EXPERIMENTAL RESULTS}

The instrumented saxophone mouthpiece is now used to inspect the strategies of an experienced player. Note that in the experiments discussed below, the FSR signal shows both a quasi-static component and an oscillating component sharing the same frequency content as the pressure signals. Since the FSR calibration has been per- 
formed only with static loads, these oscillations will not be discussed hereafter.

\section{A. Pitch bend effect : no instruction}

In this first example, the musician was asked to perform a pitch bend without any particular constraint. The starting note is $\mathrm{C} \# 5$, second register $\left(f_{0}=550 \mathrm{~Hz}\right)$.

Figure 12 shows the time variations of the three sensors signals. The mouth pressure signal shows oscillating components with the same order of magnitude than the static component and the mouthpiece pressure. A transfer of energy between the mouth and mouthpiece pressure is visible between $t=1.5 \mathrm{~s}$ and $t=3.5 \mathrm{~s}$.

The average effort on the reed is nearly constant.

Figure 13 shows the time-frequency representations (Gabor transforms) of the two pressure sensors as well as that of the external pressure. It can be noticed that the energy transfer occurs mainly on the first harmonics and is maximum when the bending effect is the most important. The frequency of the bend measured on the external pressure signal is modified from $550 \mathrm{~Hz}$ to $493 \mathrm{~Hz}$.

Figure 14 shows the Gabor mask between the mouth pressure and mouthpiece pressure. The maximum of the ratio is above 7 for the first harmonics, which means that at this particular frequency, the input impedance modulus of the vocal tract is more than seven times larger than that of the instrument. Simulation using the impedance model of equation (12) and adapted to the actual length $(\mathrm{L}=0.428 \mathrm{~m})$ of the instrument for that fingering shows that the modulus of the input impedance ratio between $550 \mathrm{~Hz}$ (close to an impedance peak) and $493 \mathrm{~Hz}$ (close to a admittance peak) is around 13. This tends to suggest that high value of the ratio on figure 14 between the mouth and mouthpiece is mainly due to the shift of the playing frequency, which falls near an impedance minimum of the instrument.

This is confirmed by the evolution of the modulus of the ratio for the second harmonics. Indeed, a $\mathrm{t}=2.5 \mathrm{~s}$, it is minimum and the simulation shows that at this frequency $(886 \mathrm{~Hz})$, the input impedance modulus of the instrument exhibits a maximum. In the same way, at $\mathrm{t}=1 \mathrm{~s}$ and $\mathrm{t}=3.5 \mathrm{~s}$, the modulus of the ratio shows a maximum for the second harmonics when crossing frequency $1100 \mathrm{~Hz}$ that is close to a minimum of the simulated input impedance of the instrument $(1165 \mathrm{~Hz})$.

\section{B. Pitch bend effect : "frozen" vocal tract}

Here, the musician was asked to perform a bend without using his vocal tract. Compared to figure 12, figure 15 shows a much more pronounced variation of the effort on the reed (the musician decreases his effort between $t=1 \mathrm{~s}$ and $t=3.5 \mathrm{~s}$, which induces an increase of the mean reed channel opening). This strategy to modify the playing frequency is not surprising and well known from saxophone players who use the same technique to perform
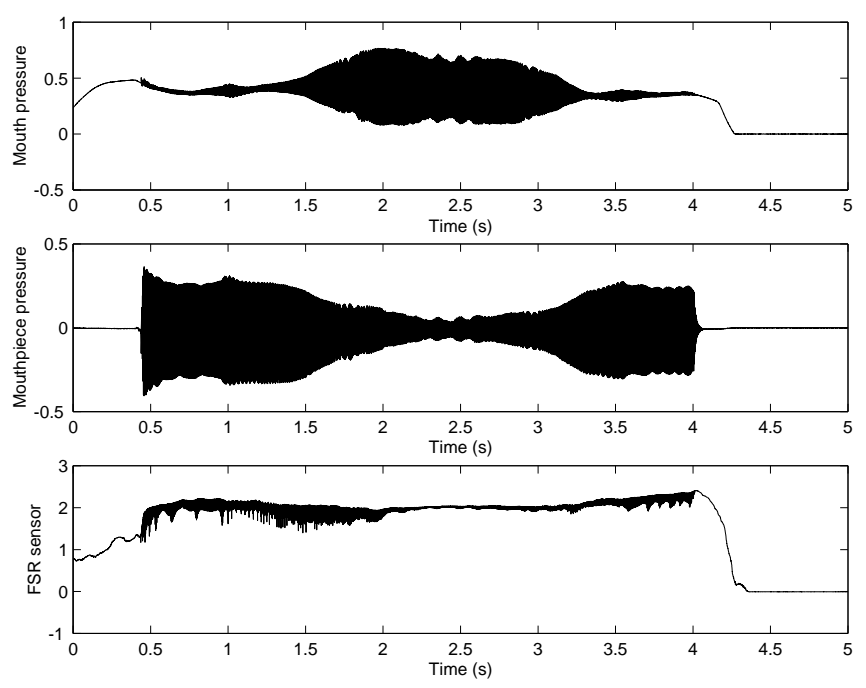

FIG. 12. Pitch bend effect : no instruction (time signals).
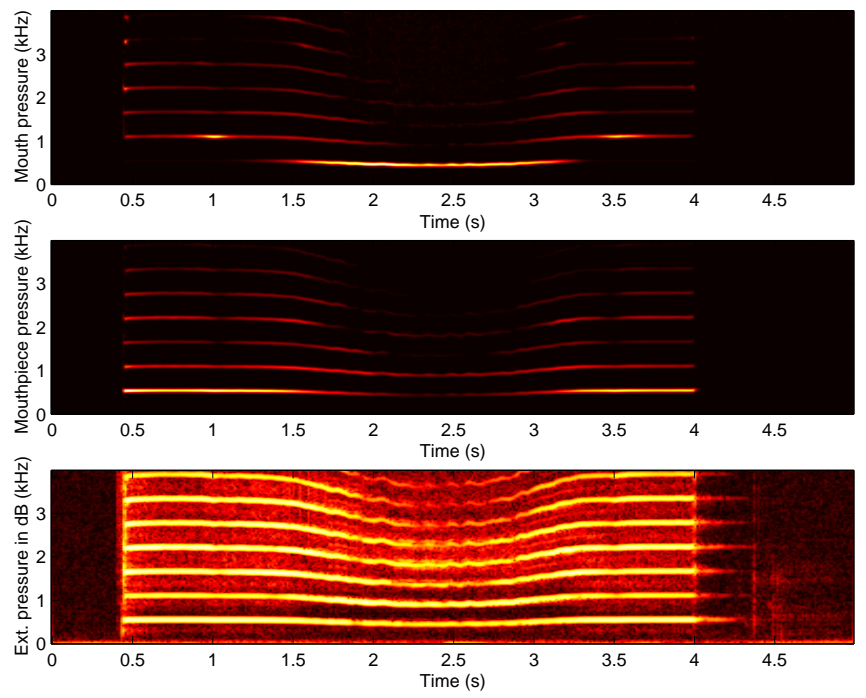

FIG. 13. Pitch bend effect : no instruction (spectrograms)

for example a vibrato ${ }^{12}$. Moreover it can be easily reproduced with time domain simulation as it can be done using the model described in ${ }^{13}$.

Compared to figure 12 , the mouth pressure shows very few oscillating components, whereas the mouthpiece pressure show less amplitude variation.

Compared to figure 13, Figure 16 shows that the strong oscillations in the mouth pressure at the frequency of the first harmonics have disappeared and that the range of the bend (measured on the external pressure signal) is much smaller than when the vocal tract is used (between $550 \mathrm{~Hz}$ and $528 \mathrm{~Hz}$ ). On the contrary, the energy is mostly concentrated around $2 \mathrm{kHz}$. Figure 17 confirms this result. 

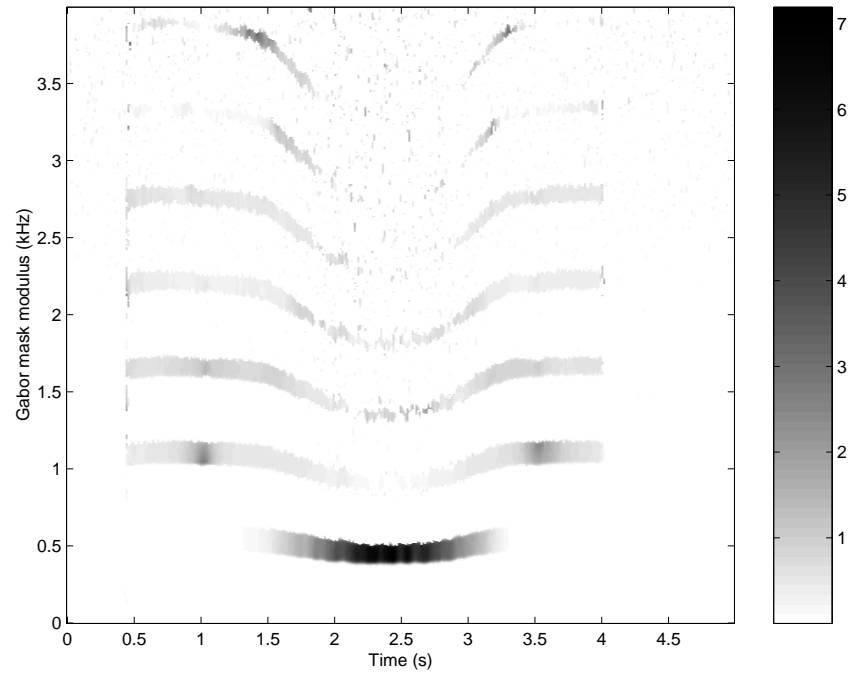

FIG. 14. Pitch bend effect : no instruction (Gabor mask)
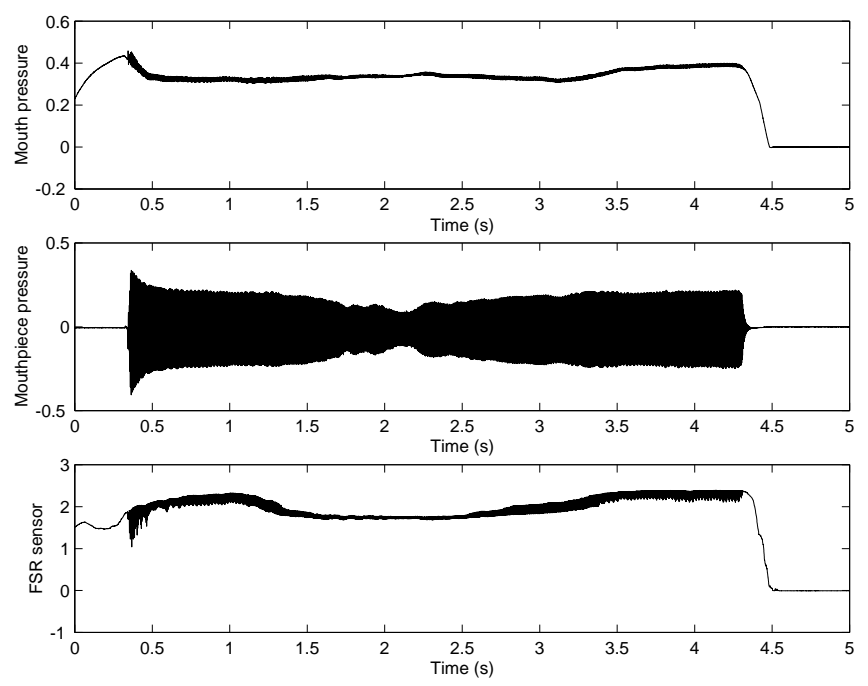

FIG. 15. Pitch bend effect : "frozen" vocal tract (time signals).

\section{Glissando}

In this example, we study the strategies developed by the player to perform a glissando. No indication is given to the player. The fingering remains constant (F3 piano, fundamental frequency $174 \mathrm{~Hz}$ ) on all the sound duration. Results are presented on figures 18, 19 and 20. The playing frequency measured on the spectrogram of the external pressure varies from $1300 \mathrm{~Hz}$ to $440 \mathrm{~Hz}$. The input impedance corresponds to that shown on figure 11. Remarkable frequencies on this figure are those of the impedance maxima, respectively : $174 \mathrm{~Hz}, 351 \mathrm{~Hz}, 538 \mathrm{~Hz}$, $735 \mathrm{~Hz}, 942 \mathrm{~Hz}$ and $1154 \mathrm{~Hz}$, and those of the impedance minima, respectively : $220 \mathrm{~Hz}, 440 \mathrm{~Hz}, 662 \mathrm{~Hz}, 885 \mathrm{~Hz}$ and $1105 \mathrm{~Hz}$.

Figure 18 shows that the lip pressure remains remarkably stable until $\mathrm{t}=5.8 \mathrm{~s}$. At this point the amplitude of
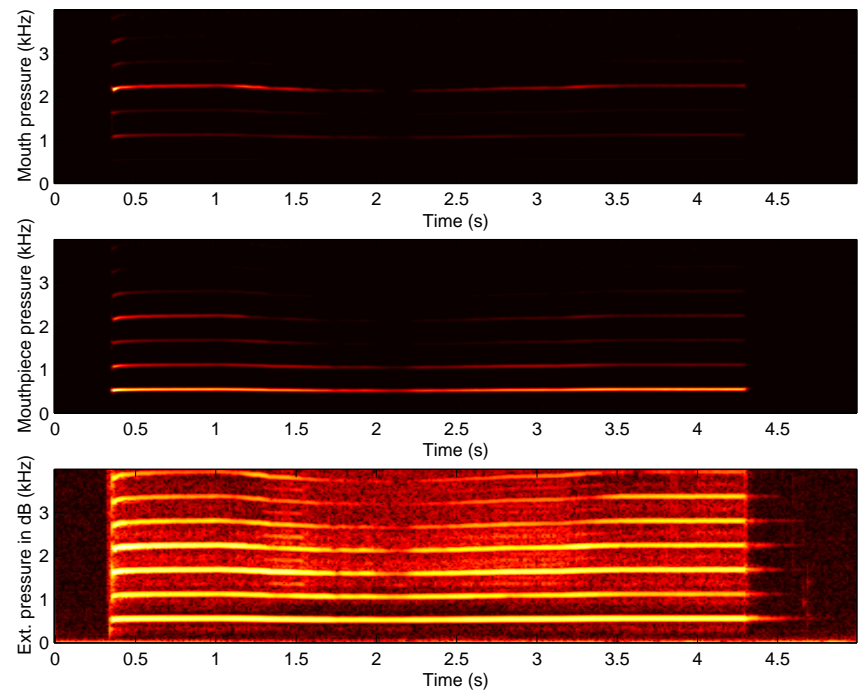

FIG. 16. Pitch bend effect : "frozen" vocal tract (spectrograms)

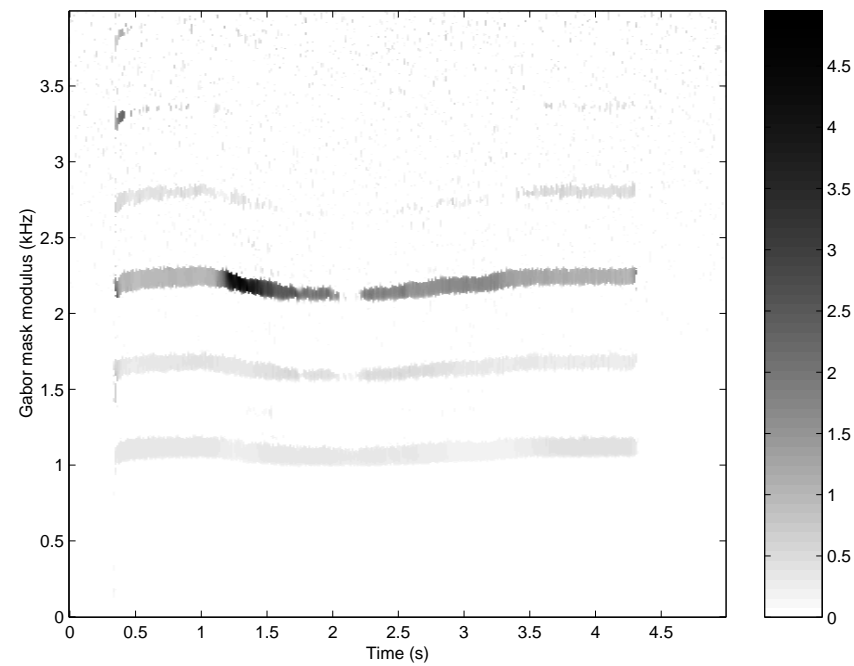

FIG. 17. Pitch bend effect : "frozen" vocal tract (Gabor mask)

both mouth and mouthpiece pressures increase. Figure 19 shows that at this point the glissando exhibits a frequency jump. This jump is not wished by the payer and corresponds to a loose of control. It can be explained by figure 20 which shows that the impedances ratio at the fundamental frequency varies very quickly from a maximum around $\mathrm{t}=5 \mathrm{~s}(650 \mathrm{~Hz})$ to a minimum at $t=5.8 \mathrm{~s}$ $(540 \mathrm{~Hz})$. These frequencies correspond respectively, with a very good agreement, to the frequencies of the third minimum and maximum of the saxophone impedance model. This phenomena is also observed on the same figure from the beginning of the glissando, with a modulation in time of the amplitude of the impedance ratio at the fundamental frequency, with maxima at frequencies very close to those of the saxophone impedance minima and minima very close to those of the saxophone impedance 

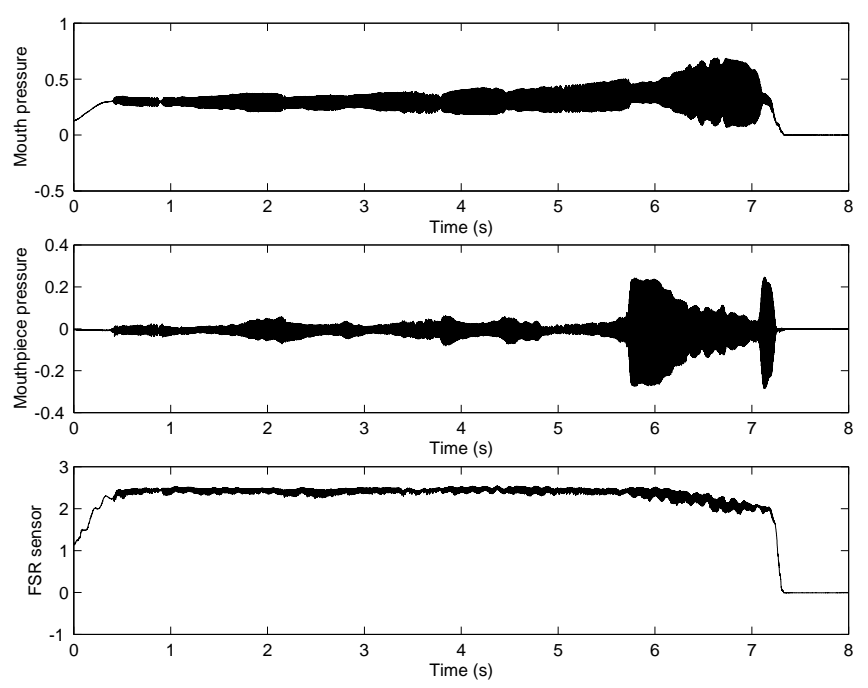

FIG. 18. Glissando (time signals).

maxima. It can be noticed that maxima and minima are less pronounced at high frequency than at low frequency, as well as the frequency jumps. This can be explained by the fact that at high frequencies, both impedance maxima and minima are less pronounced that at low frequencies, as shown on figure 11.

This shows that the glissando is achieved by the musician with a functioning of the whole system on a sliding impedance peak of its vocal tract, while the instrument can be considered as a perturbation whose disturbing effect is more important as the playing frequency decreases. Indeed until $\mathrm{t}=5.8 \mathrm{~s}$, the impedance ratio remains always bigger than one. The sudden change in the mouthpiece pressure amplitude at $t=5.8 \mathrm{~s}$ indicates that the functioning of the system is driven by the third impedance peak of the instrument, the frequency of the oscillation is then modified by the vocal tract influence. The very fast vibrato observed on the external pressure indicates that this modification is difficult to achieve for the musician. Moreover, at $\mathrm{t}=7 \mathrm{~s}$, the disturbing effect becomes to strong and the functioning of the system gets back to a normal mode where the playing frequency is imposed by the second impedance peak of the saxophone $(350 \mathrm{~Hz})$. The transition is clearly visible on figure 19 .

\section{Chromatic scale : legato}

In this example, the musician is asked to perform a legato chromatic scale in the first register without any indication (figures 21, 22, 23) and without using his vocal tract (figures 24, 25, 26).

When the vocal tract is not used, the lip pressure varies more than when it is used, which shows that the musicians compensates for a lack. This adaptation is different for each note. It can be observed that in the upper half of the first register, the lip pressure is increased slightly with the note played. This effect is more pronounced when the
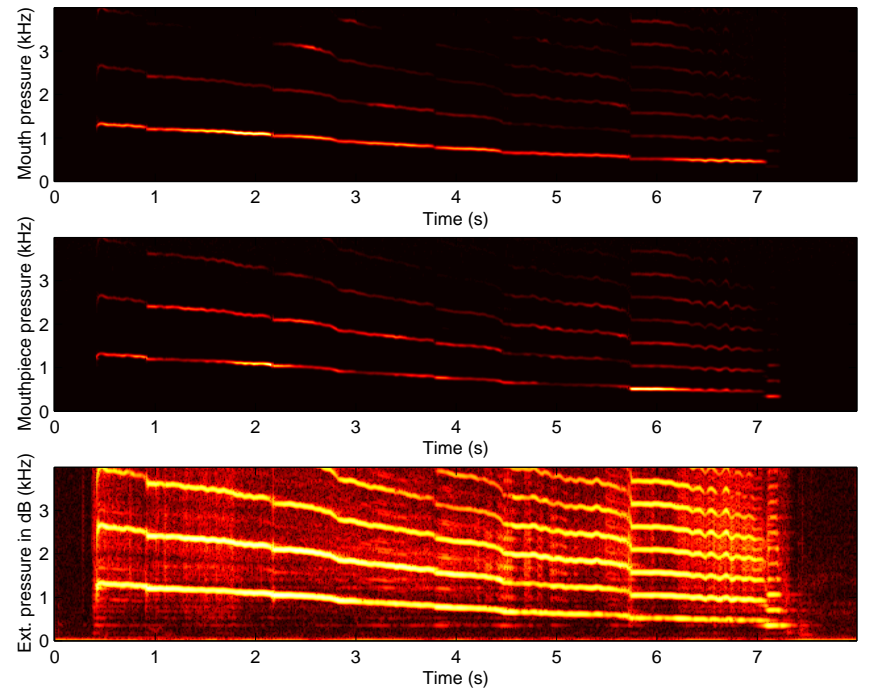

FIG. 19. Glissando (spectrograms).

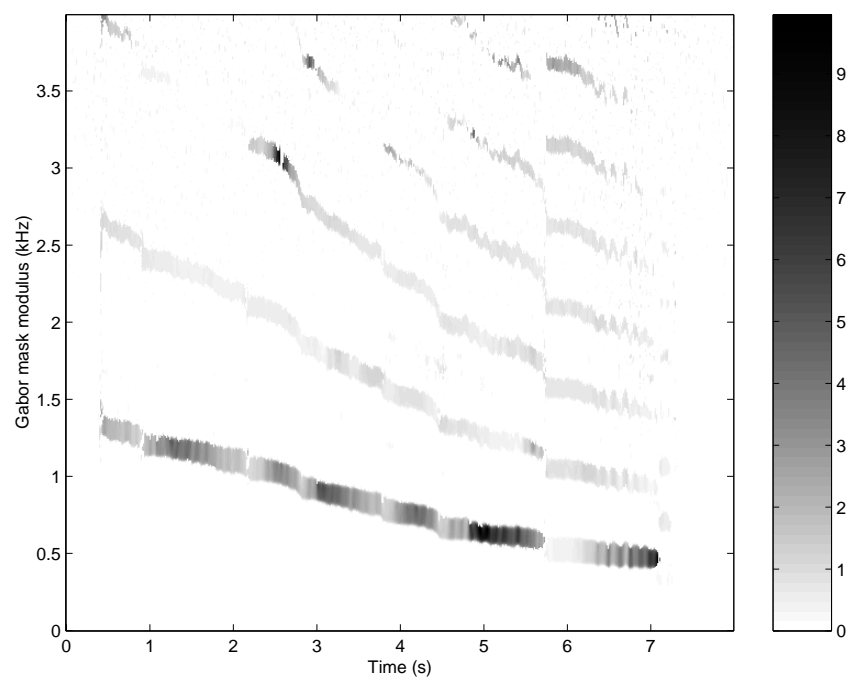

FIG. 20. Glissando (Gabor mask).

vocal tract is not used. When the use of the vocal tract is not forbidden, the oscillations of the mouth pressure are less pronounced, particularly in the second part of the first register. A close look at figures 22 and 25 would reveal that for each note, the frequency shift between the beginning of the note and its steady state is more important when the vocal tract is not used. Therefore, with the help of the vocal tract, the right pitch seems easier to reach, as it can be seen on the external pressure spectrogram. When the vocal tract is used, the modulus of the impedances ratio remains smaller for frequencies below $1 \mathrm{kHz}$ whatever the note played. On the contrary, when the vocal tract is not used, it can be seen on figure 26 that for notes between $6 \mathrm{~s}$ and $8 \mathrm{~s}$, the ratio reaches significantly high values for the third harmonics. This corresponds to a frequency band between $700 \mathrm{~Hz}$ and $900 \mathrm{~Hz}$. Similarly, 

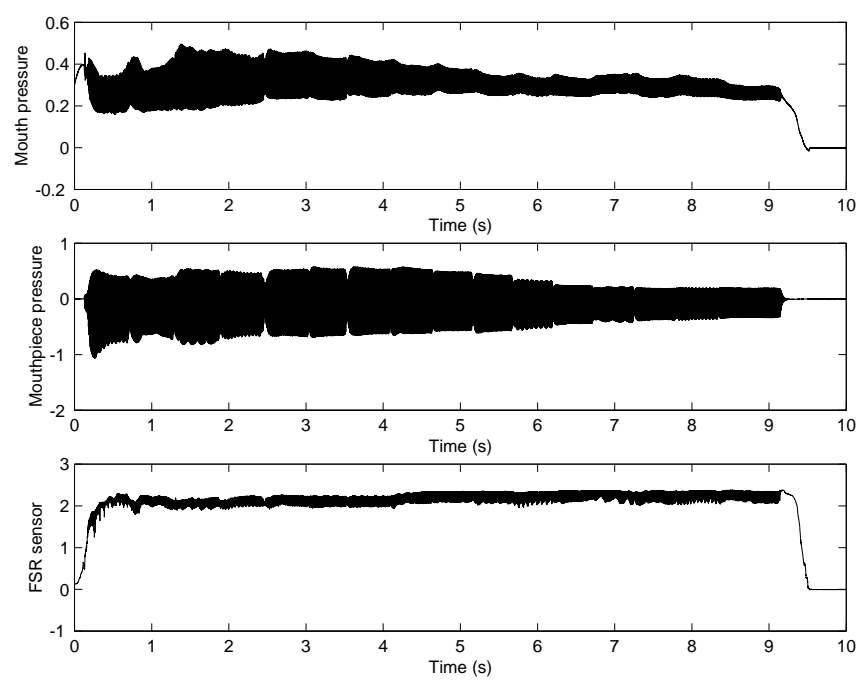

FIG. 21. Chromatic scale : legato, unconstrained (time signals).

within this band, between $\mathrm{t}=3 \mathrm{~s}$ and $\mathrm{t}=5 \mathrm{~s}$, the ratio reach high values for the fourth harmonics of the note played. For these notes, the mouth pressure becomes maximum within this frequency band (see figure 25, top).

Impedances calculations for several bore lengths using formula (12) show that this maximum of mouth pressure is not due a specific resonance of the vocal tract but corresponds to an antiformant of the mouthpiece pressure, as it has been explained in ${ }^{6}$. For notes between $6 \mathrm{~s}$ and $8 \mathrm{~s}$, a close look at figure 25 reveals that the correct pitch is the most difficult to obtain (pitch instability). Here, the strategy of the musician when using his vocal tract seems to be the compensation of the antiformant of the mouthpiece pressure in order to avoid a high level of oscillations in his mouth. This compensation seems to be the decrease of the vocal tract impedance at these frequencies. The result observed on the external pressure is an increased playability of the notes.

\section{E. Chromatic scale : attacks}

This example deals with chromatic scales in the first and second registers, with detached notes produced by a tongue attack. It is intended to study the strategy of the musician to produce neat attacks and obtain the right pitch. Figure 27 shows that, until the ninth note of the first register, the musician nearly totally releases his lip pressure on the reed before the birth of each note, probably in order to be able to produce the proper note of the first register without jumping to the second one. Indeed, as shown in ${ }^{17}$, the linear stability analysis reveals that for large lip pressures, the first oscillation threshold corresponds to the second register. Moreover, it is well known even from beginners that attacks on the first register can only be achieved by releasing the lip pressure.

The lip pressure profile is similar for all the notes of
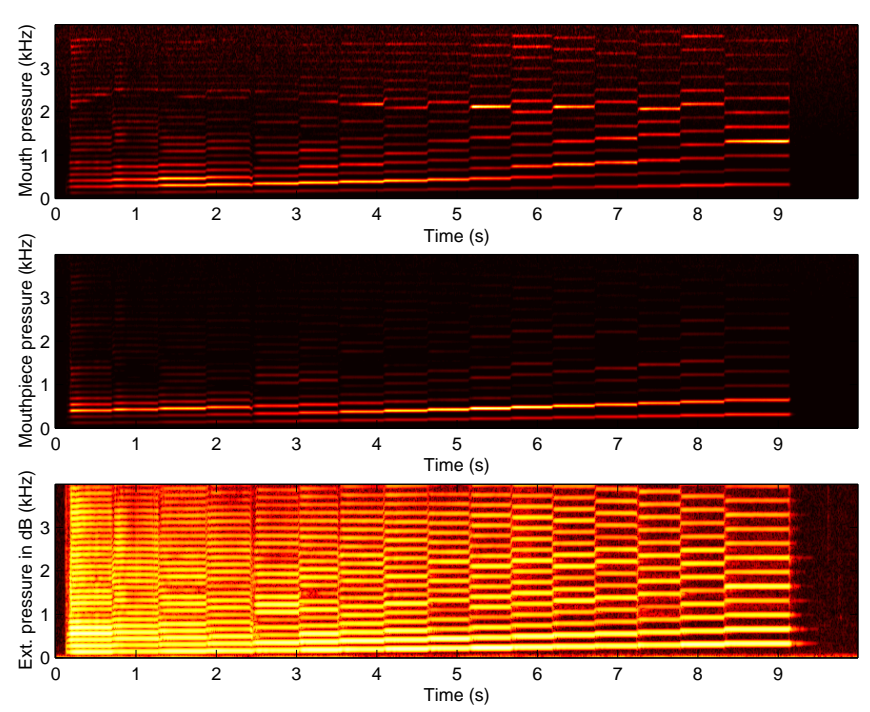

FIG. 22. Chromatic scale : legato, unconstrained (spectrograms).

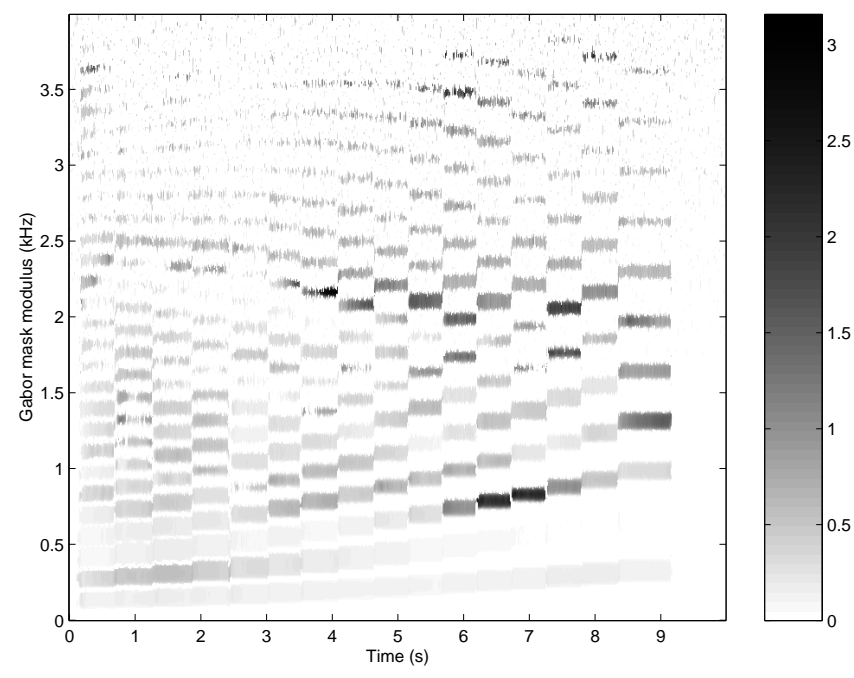

FIG. 23. Chromatic scale : legato, unconstrained (Gabor mask).

both the first and the second register (figures 27 and 28) with a fast increase during the transient. It can be noticed that in the first register, the maximum lip pressure is increasing from note to note, as well as the mean value. It can also be noticed that in the second register (see figure 28), the increase of the lip pressure with respect to the note played is less important than in the first register but that the average lip pressure is still more important (probably to avoid to skip back on the first register).

A close look at one of the notes of the second register (see figure 29) shows that both the attack and release time (the time to reach a steady-state) of the mouth pressure are smaller than those of the mouthpiece pressure. This is particularly visible on the release. This can be explained by the fact that the quality factors of the im- 

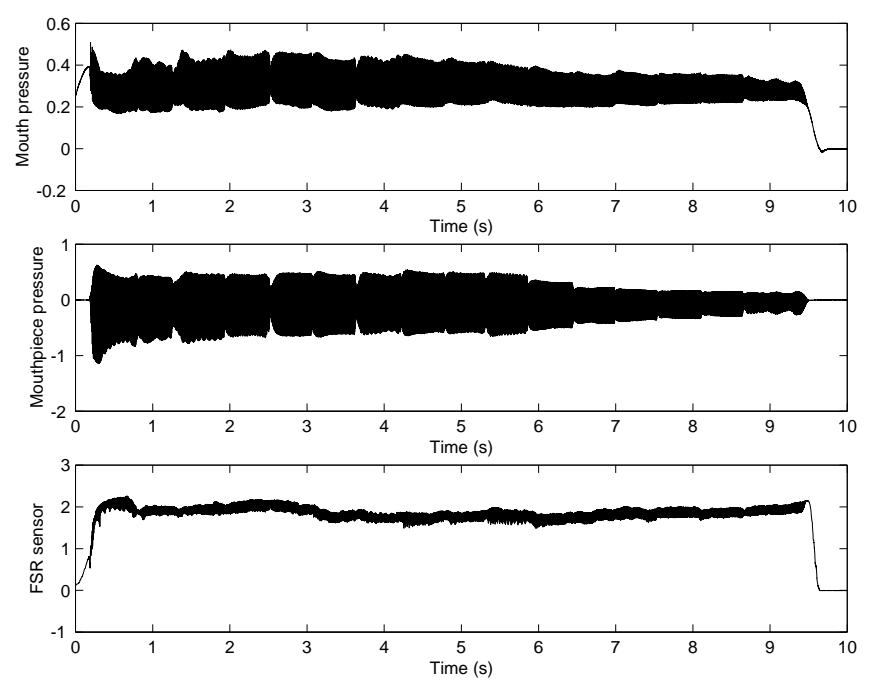

FIG. 24. Chromatic scale : legato, "frozen" vocal tract (time signals).
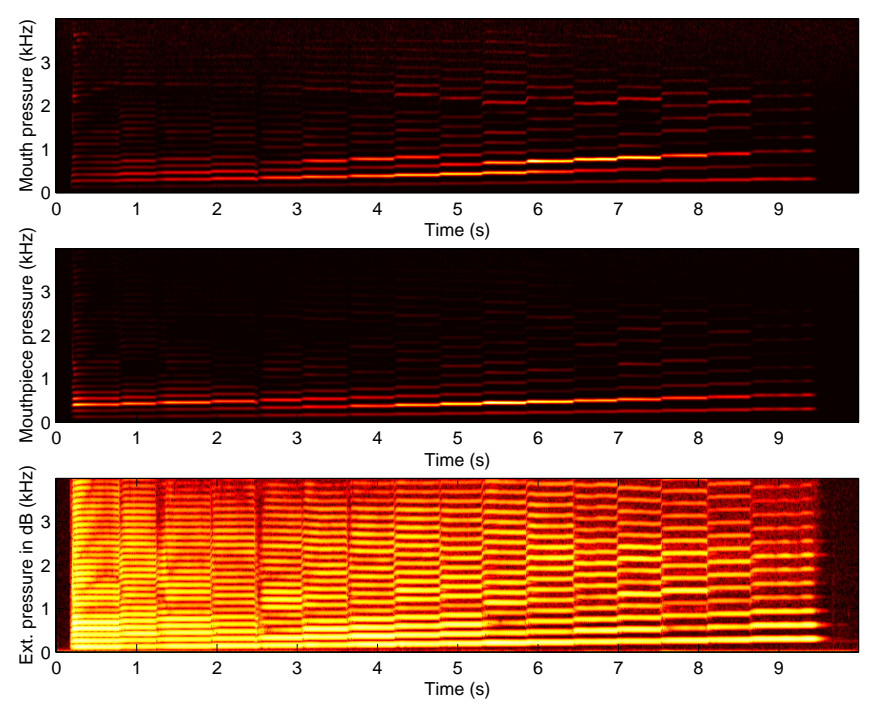

FIG. 25. Chromatic scale : legato, "frozen" vocal tract (spectrograms).

pedance peaks of the saxophone are much larger than those of the vocal tract. On the mouth pressure, it can be seen that the tongue removal leads to a drop of the quasi-static pressure during all the note duration (figure 29, top). The lip pressure exhibits a typical shape, with first a fast increase until $\mathrm{t}=0.07 \mathrm{~s}$ followed by a slower increase until $t=0.2 s$ corresponding to the adaptation of the musician to obtain the proper pitch. This is due to the fact that the more the reed channel is open, the more the playing frequency is lower than that of the second input impedance peak of the instrument. This is confirmed by the top of figure 30 which shows the variations of the playing frequency during the note. It increases until $\mathrm{t}=0.15 \mathrm{~s}$ and remains stable until $\mathrm{t}=0.25 \mathrm{~s}$, following the variations of the lip pressure. After $\mathrm{t}=0.25 \mathrm{~s}$, the fre-

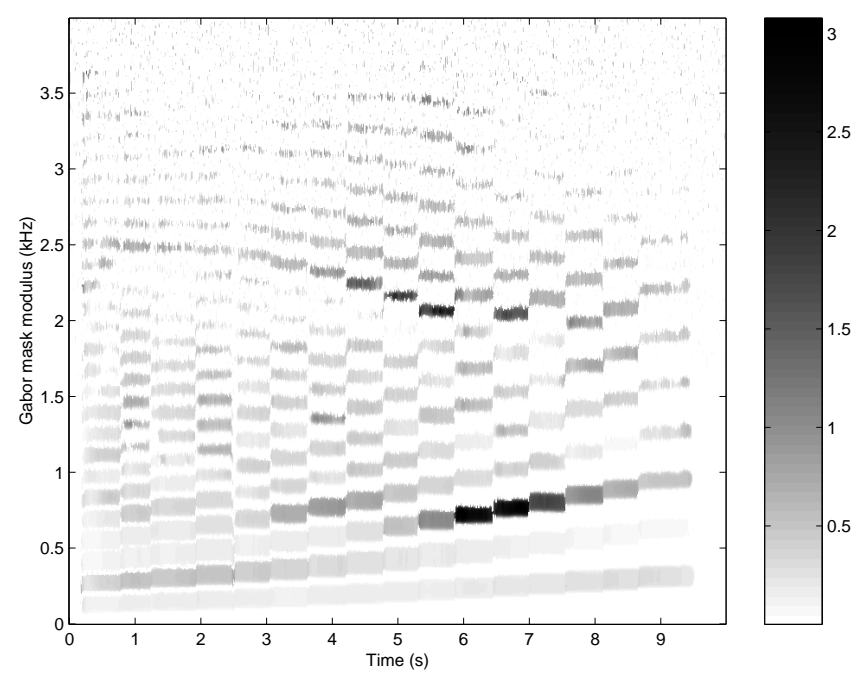

FIG. 26. Chromatic scale : legato, "frozen" vocal tract (Gabor mask).
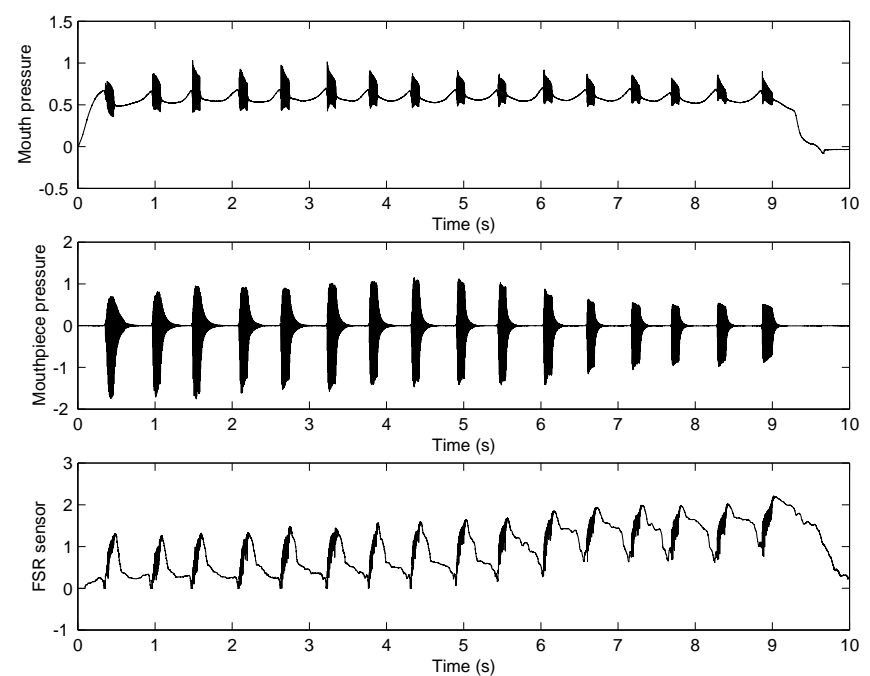

FIG. 27. Chromatic scale : attacks, first register (time signals).

quency increases suddenly while the mouthpiece pressure starts to decrease exponentially. This corresponds to free oscillations of the air column of the instrument once the tongue closes the reed channel, with an oscillation frequency and release time corresponding to that of the second impedance peak of the saxophone.

\section{CONCLUSIONS}

In this study, it has been shown that the control of the musician on his instrument can be very subtile. In particular, the use of the vocal tract reinforce or makes possible some particular regimes (pitch bend, glissando), which confirms results previously obtained ${ }^{18}$. Moreover, it has been shown in this paper that in some cases the vocal tract influence is so important that the functioning of 

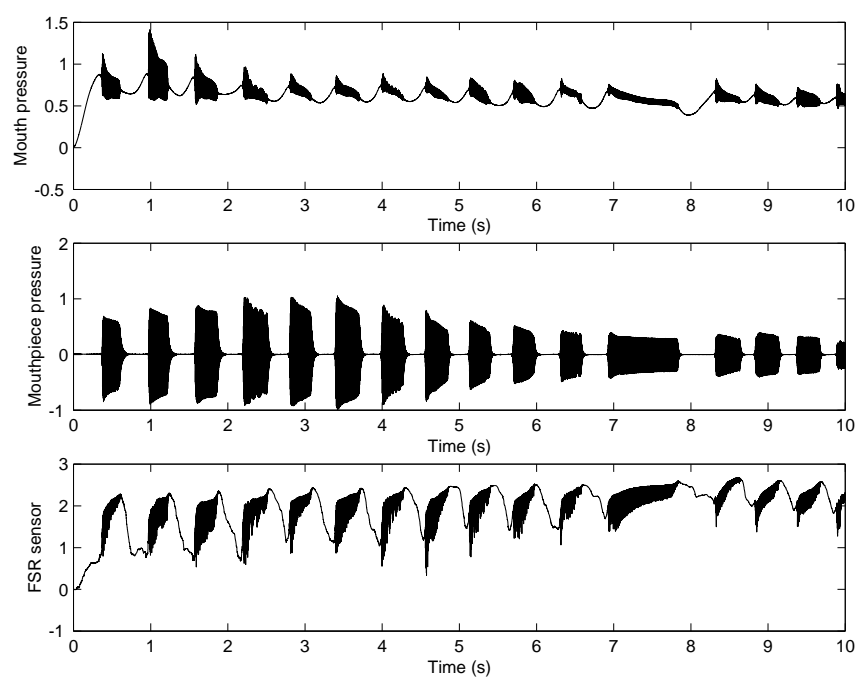

FIG. 28. Chromatic scale : attacks, second register (time signals).
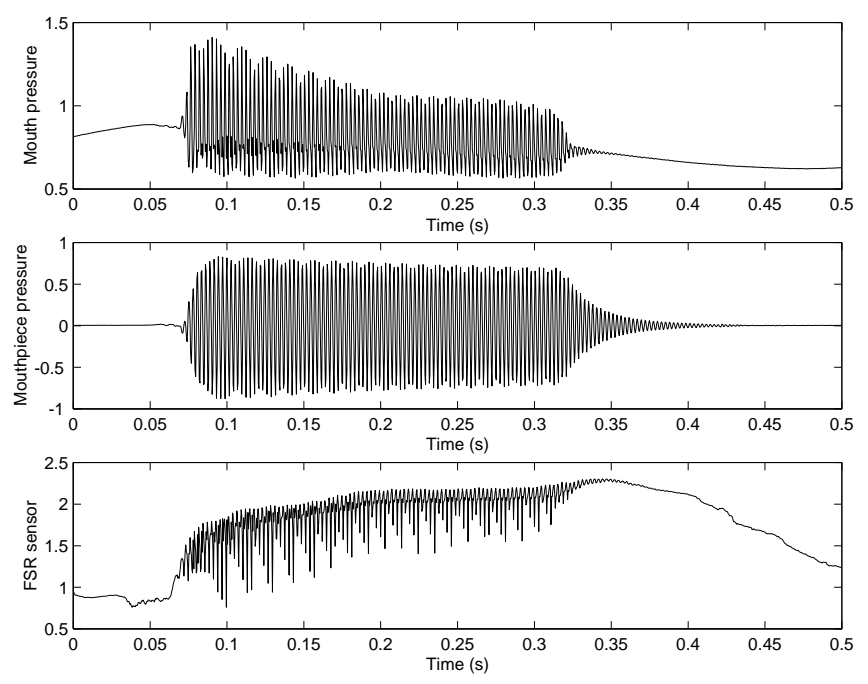

Fig. 29. Zoom on an attack, second register (time signals).

the whole system can be nearly totally driven by it even at frequencies corresponding to an impedance minimum of the saxophone. On a more standard play, the musician is capable to minimize the role of his vocal tract so that it does not disturb the functioning of the instrument ${ }^{20}$. The simple device that has been developed, even if no quantitative information is given about the reed channel opening, makes it possible to study the way the musician uses his lip pressure to control the pitch and the regime selection during the play. This provides informations on how physical models should be controlled for expressive sound synthesis and how artificial mouthes should be piloted in order to reproduce natural tongue attacks.

Perspectives of this study are concerned with the understanding and the exploitation of the oscillating component of the FSR signal that may contain informations
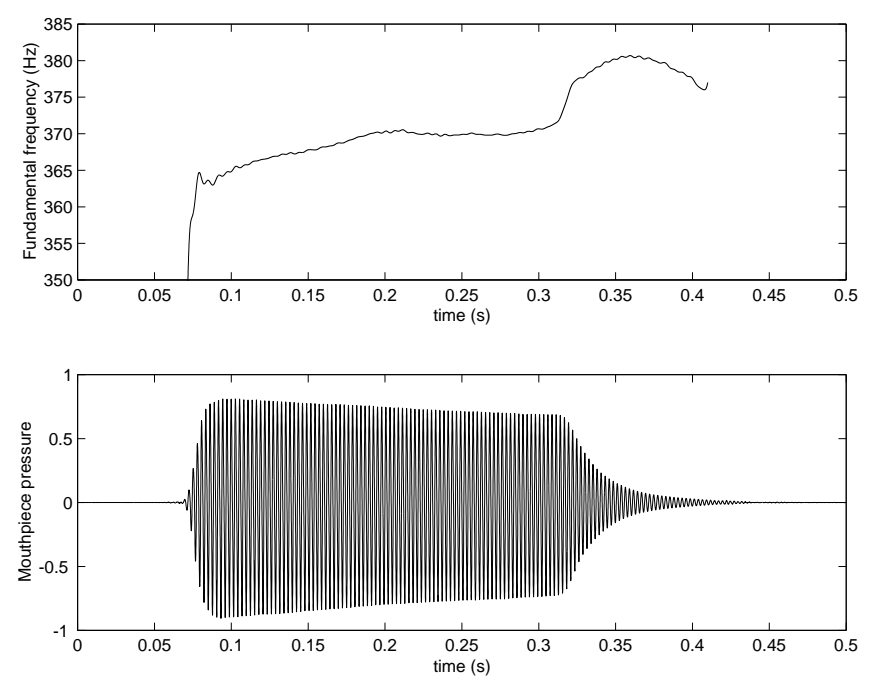

FIG. 30. From the data of figure 29, instantaneous frequency synchronized with the external pressure.

on mechanical properties of the reed and how they vary with respect to the lip pressure, and with an automated control of vocal tract models used for real-time sound synthesis ${ }^{14}$ in order to improve the playability.

\section{ACKNOWLEDGMENTS}

The authors would like to thank S. Karkar and J. Kergomard for fruitful discussions, as well as musicians from CEFEDEM, Aubagne. The study presented in this paper was lead with the support of the French National Research Agency ANR within the CONSONNES project.

${ }^{1} \mathrm{~J}$. Backus, "The effect of the player's vocal tract on woodwind instrument tone", J. Acoust. Soc. Am., 78(1), 17-20 (1985).

${ }^{2}$ A. Benade, P. Hoekje, "Vocal tract effects in wind instrument regeneration", J. Acoust. Soc. Am. Suppl, 71(1), 591 (1985).

${ }^{3}$ Rene Causse, Vincent Freour, "Evaluation of two control parameters of trumpet players as function of sound features. (A)", J. Acoust. Soc. Am. Volume 125, Issue 4, pp. 2597-2597

${ }^{4} \mathrm{P}$. Clinch, G. Troup, L. Harris, "The importance of the vocal tract resonance in clarinet and saxophone performance : A preliminary account", Acustica, Vol. 50, 280-284, (1982).

${ }^{5}$ P. de la Cuadra, B. Fabre, N. Montgermont, C. Chafe, "Analysis of Flute Control Parameters : A Comparison Between a Novice and an Experienced Flautist", Acta Acustica united with Acustica, Volume 94, Number 5, pp. 740-749(10) (2008)

${ }^{6}$ J. P. Dalmont, B. Gazengel, J. Kergomard, "Scaling of reed instruments : The case of the saxophone family (A)", J. Acoust. Soc. Am. Volume 119, Issue 5, pp. 3259-3259 (2006)

${ }^{7}$ M. Demoucron, A. Askenfelt, R. Causse, "Measuring Bow Force in Bowed String Performance : Theory and Implementation of a Bow Force Sensor", Acta Acustica united with Acustica, Volume 95, Number 4, pp. 718-732(15) (2009)

${ }^{8}$ P. Depalle, R. Kronland-Martinet, B. Torresani, "Time-frequency multipliers for sound synthesis", SPIE annual Symposium Wavelet XII, San Diego : United States (2007), DOI : $10.1117 / 12.732447$

${ }^{9}$ H. G. Feichtinger, K. Nowak. "A first survey of Gabor multipliers", In H. G. Feichtinger and T. Strohmer, editors, Advances in Gabor Analysis, Boston, 2002. Birkhauser. 
${ }^{10} \mathrm{C}$. Fritz, "La clarinette et le clarinettiste : influence du conduit vocal sur la production du son" (The clarinet and the player : role of the vocal tract on the sound production), $\mathrm{Ph}$. D. thesis, Univ. Paris 6 and New South Wales, France (2005).

${ }^{11}$ L. Fuks, J. Sundberg, "Blowing Pressures in Bassoon, Clarinet, Oboe and Saxophone", Acta Acustica united with Acustica, Volume 85, Number 2, pp. 267-277(11) (1999)

12 J. Gilbert, L. Simon, and J. Terroir, "Vibrato of saxophones", J. Acoust. Soc. Am. Volume 118, Issue 4, pp. 2649-2655

${ }^{13} \mathrm{P}$. Guillemain, J. Kergomard, T. Voinier, "Real-time synthesis of clarinet-like instruments using digital impedance models", J. Acout. Soc. Am, Vol. 118(1), 483-494, (2005).

${ }^{14} \mathrm{Ph}$. Guillemain, "Some roles of the vocal tract in clarinet breath attacks : Natural sounds analysis and model-based synthesis", J. Acoust. Soc. Am, Vol. 121(4), pp. 2396-2406 (2007).

${ }^{15} \mathrm{P}$. Guillemain, R. Kronland-Martinet, "Characterization of acoustic signals through continuous linear time-frequency representations", Proc. of the IEEE, Vol. 84 (4), pp. 561-585, (1996)
${ }^{16}$ D. H. Keefe, "Acoustical wave propagation : transmission line parameter approximations for isothermal and non isothermal boundary conditions", J. Acous. Soc. Am. , 75(1), pp 58-62, (1984)

${ }^{17}$ B. Ricaud, Ph. Guillemain, K. Kergomard, F. Silva, C. Vergez, "Behaviour of reed woodwind instruments around the oscillation threshold", Acta Acustica united with Acustica, Volume 95, Number 4, pp. 733-743(11) (2009)

${ }^{18}$ G. Scavone, A. Lefebvre, A. R. da Silva, "Measurement of vocaltract influence during saxophone performance", J. Acoust. Soc. Am. Volume 123, Issue 4, pp. 2391-2400 (2008).

${ }^{19}$ A. Tarnopolsky, N. Fletcher, L. Hollenberg, B.Lange, J. Smith, J. Wolfe, "Acoustics : The vocal tract and the sound of a didgeridoo", Nature 436, 39 (2005)

${ }^{20} \mathrm{~T}$. Wilson, "The measured vocal tract impedance for clarinet performance and its role in sound production", J. Acoust. Soc. Am, 99(4), 2455-2456, (1996). 